\title{
Preliminary Measurements of the Motion of Arcjet Current Channel Using Inductive Magnetic Probes
}

\author{
Magnus A. Haw*, \\ Analytical Mechanics Associates, Moffett Field, CA, 94035 \\ Jeremie B. E. Meurisse ${ }^{\dagger}$ \\ Science and Technology Corporation, Moffett Field, CA, 94035 \\ Sander Visser ${ }^{\ddagger}$, Sergio F. Izquierdo ${ }^{\S}$, \\ University of Illinois at Urbana-Champaign, Urbana, IL, 61801 \\ Joseph C. Schulz ${ }^{\text {I }}$ \\ Analytical Mechanics Associates, Moffett Field, CA, 94035 \\ Nagi N. Mansour \| \\ NASA Ames Research Center, Moffett Field, CA 94035
}

\begin{abstract}
This paper covers the design and first measurements of non-perturbative, external inductive magnetic diagnostics for arcjet constrictors which can measure the motion of the arc currentchannel. These measurements of arc motion are motivated by previous simulations using the ARC Heater Simulator (ARCHeS), which predicted unsteady arc motion due to the magnetic kink instability. Measurements of the kink instability are relevant to characterizing motion of the enthalpy profile of the arcjet, the arcjet operational stability, and electrode damage due to associated arc detachment events. These first measurements indicate $\pm 4 \mathrm{~mm}$ oscillations at $0.5-2 \mathrm{kHz}$ of the current profile.
\end{abstract}

\section{Nomenclature}

$\begin{array}{ll}\mathrm{ARCHeS} & =\text { ARC Heater Simulator } \\ \mathrm{AHF} & =\text { Aerodynamic Heating Facility } \\ \text { MHD } & =\text { magnetohydrodynamic } \\ \mathbf{B} & =\text { magnetic field vector } \\ \bar{B} & =\text { average magnetic field magnitude } \\ I & =\text { electric current } \\ \Phi & =\text { magnetic flux } \\ \delta & =\text { radial displacement } \\ \omega & =\text { angular frequency } \\ V_{\text {emf }} & =\text { induced electromotive force }\end{array}$

\section{Introduction}

THOUgh the plume and nozzle region of arcjets have been extensively diagnosed, the constrictor or column region Aremains relatively unmeasured due to the harsh environment. Similar environments in space and fusion devices

\footnotetext{
*Plasma physicist, Thermophysics Materials Branch, NASA Ames Research Center, MS 234-4, Member AIAA, magnus.haw@nasa.gov

$\dagger$ Aerospace Engineer, NASA Advanced Supercomputing Division, Moffett Field

${ }^{\ddagger}$ Research Scholar, NASA Advanced Supercomputing Division, Moffett Field

$\S$ Research Scholar, NASA Advanced Supercomputing Division, Moffett Field

IIAerospace Engineer, NASA Advanced Supercomputing Division, Moffett Field

"Chief Division Scientist, NASA Advanced Supercomputing Division, Moffett Field
} 


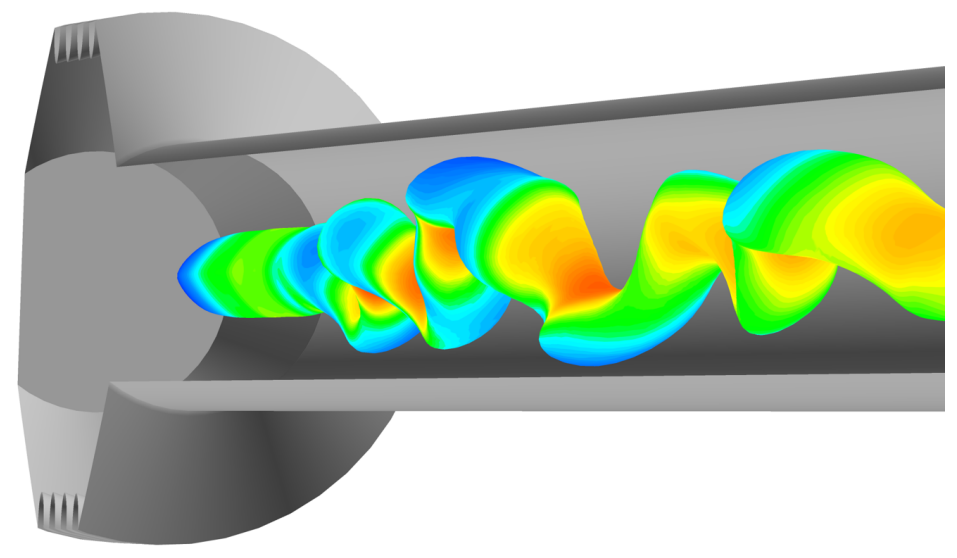

Fig. 1 Simulated current path from ARCHeS showing twisted current path inside the constrictor region.

require either disposable or non-intrusive diagnostics. This work will describe the development and first measurements of external magnetic diagnostics which provide a non-perturbative, sensitive measurement of the arc current-channel motion.

These measurements were motivated by simulation results from a new software tool, the ARC Heater Simulator (ARCHeS), a 3D radiative magnetohydrodynamic (MHD) code which is being developed to better understand the physics and operation of constricted arc jets [1]. Preliminary simulations using ARCHeS show the development of the magnetic kink instability in the arc column. The kink instability perturbs the current in a helical manner such that the current follows a corkscrew-like path and rotates [2, 3]. This radial displacement of the current path produces an oscillating magnetic field that can be measured outside the column. The kink instability is of particular interest because it can be easily measured and, if present, would demonstrate that magnetic forces affect mixing and unsteady flow in the column region. This has several important implications:

1) Magnetic asymmetries destabilize the flow in the column

2) Magnetic forces are likely important drivers of arc detachment events

3) External magnetic fields could suppress detachment events thereby reducing associated electrode damage, and increasing the stable operational parameter space.

\section{Inductive Magnetic Probes}

Inductive magnetic sensors are coils of wire which measure the time derivative of the magnetic field $\dot{B}$ : when the linked magnetic flux $\Phi=N \int B d A \approx N A B$ changes in time, it induces a voltage $V_{\text {emf }}$ across the coil,

$$
\begin{aligned}
V_{e m f} & =\frac{d \Phi}{d t} \\
& \approx N A \frac{d B}{d t}
\end{aligned}
$$

where $N$ is the number of turns and $A$ is the coil area. For this reason, inductive probes are also referred to as B-dot probes. This is ideal for this application since internal motion of the current path will only change the total magnetic field by $|\Delta B|<1 \mathrm{G}$ but $|\dot{B}| \sim 10^{4} \mathrm{G} / \mathrm{s}$.

These sensors are advantageous for several reasons: probes can be mounted outside the column so no high temperature materials or active cooling systems are needed, probes do not perturb the column flow/heating, probes are simple, cheap, and robust, and probe parameters/shape can be quickly iterated to optimize a given application. This flexibility in sensitivity/shape is further leveraged by using 3D printing for the coil spools and mounts.

\section{A. Optical Link Circuit}

An optical data link is necessary to prevent risk from high voltage shocks from the arcjet. This optical isolation allows the coil to be placed directly on the column and charge to arbitrary floating voltages without risk to personnel or 
digitization devices. The specifications of the optical link also inform the coil design (e.g., $\mathrm{min} / \mathrm{max}$ voltages, time response).

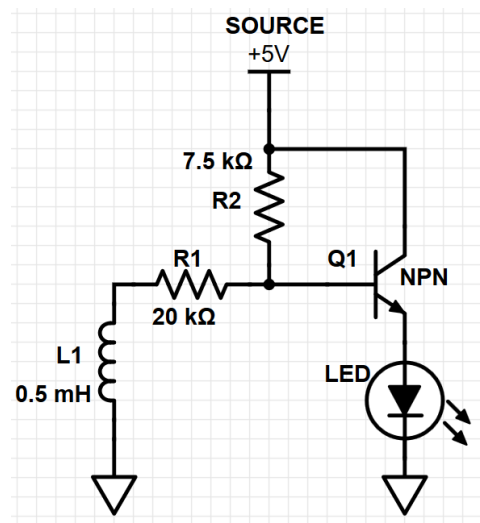

Fig. 2 Optical transmitter circuit diagram: L1 is the inductor coil shown in Figure 5 . The inductor modulates the current at the transistor (Q1) base which is amplified through the LED (HFBR-1414).

The optical link circuit uses a powered infrared LED/photodiode pair (HFBR-1414/HFBR-2416). These parts use the ST optical fiber connector. The basic optical transmitter circuit has been adapted from [4] and is shown in Figure 2 This is a simple current amplifier powered by a $5 \mathrm{~V}$ power supply: the current passing through the inductive coil L1 modulates the transistor gate, sending an amplified signal through the LED. The LED is always lit such that negative and positive fluctuations on the gate can be linearly captured as dimming and brightening respectively. The LED transmits the optical signal through a fiber to the receiver module.

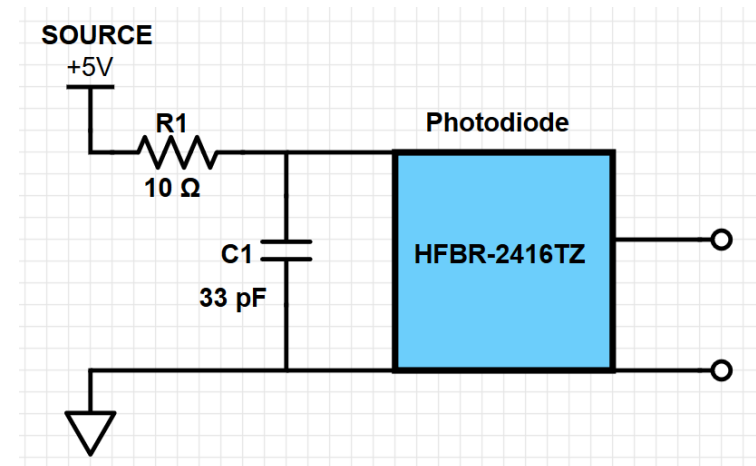

Fig. 3 Optical receiver circuit using active photodiode (HFBR-2416).

The optical receiver uses an active (reverse-biased) photodiode which converts the optical signal back into a voltage for the oscilloscope to measure. Figure 3 shows this circuit. Optionally, to remove the positive offset from the LED, a capacitor can be added in series to the receiver output. In the arcjet tests, this offset was removed using the AC coupling setting of the oscilloscope.

The 5V supply for the optical transmitter is powered by batteries to ensure full electrical isolation. The circuit uses $9 \mathrm{~V}$ batteries and a basic $5 \mathrm{~V}$ regulator (LM340MP) to provide the $5 \mathrm{~V}$ power supply. An additional triple throw switch attached to a zener diode is added to provide a simple test for battery charge: if the battery voltage is above $7.7 \mathrm{~V}$, the LED will light up.

\section{B. Version 1: Large single coil}

The first iteration was designed to determine the strength of the magnetic signal and inform the design of later iterations. The coil was made from 50 turns of gauge 33 magnet wire wrapped around a 3D printed mount (PLA material, coil dimensions: $4.2 \times 6 \times 1 \mathrm{~cm}, L=0.24 \mathrm{mH}$ ). A layer of rubber tape was placed around the coil for additional 


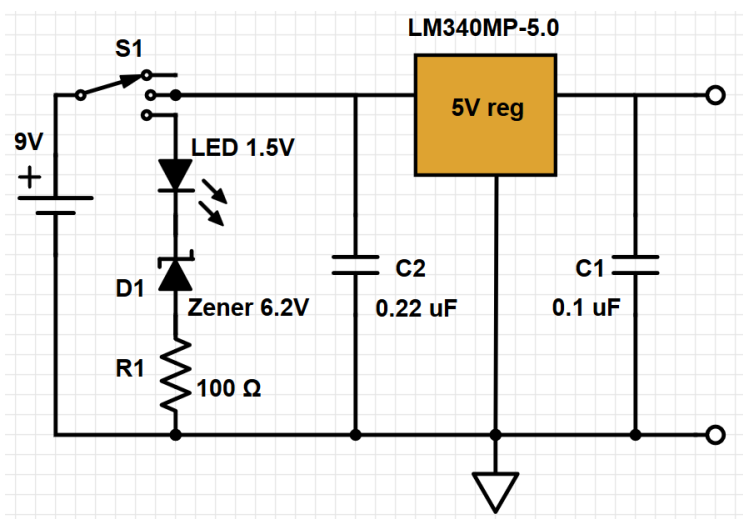

Fig. 4 Circuit diagram for 5V battery power supply. Standard voltage regulator circuit with an additional switch position to determine if $9 \mathrm{~V}$ battery has sufficient charge.

electrical insulation. The mount was designed to rest on the red plastic beam below the arcjet between packs $5 \& 6$ as shown in Figure 5 .

$$
\begin{aligned}
& N=50 \\
& W=6 \mathrm{~cm} \\
& L=6 \mathrm{~cm} \\
& H=4.2 \mathrm{~cm} \\
& \text { Gauge } 30 \\
& \text { magnet wire }
\end{aligned}
$$

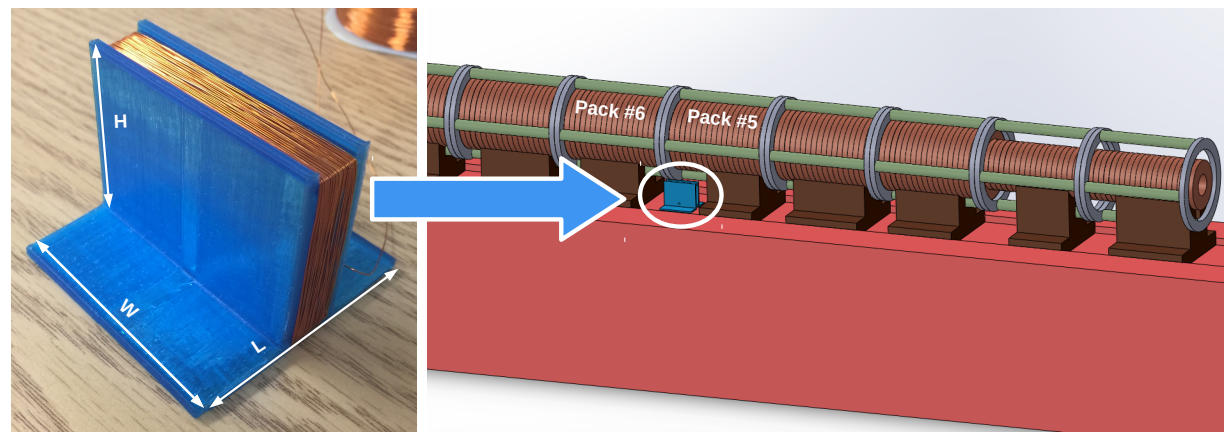

Fig. 5 Image of inductive coil sensor and coil placement below AHF heating column

The free leads of the coil magnet wire was used to connect the coil to the optical link circuit. This created two problems:

1) The fragile magnet wire was prone to breaking under movement/adjustment of the coil

2) The optical link circuit needed to be placed very close to the coil since the free magnet wire only extended $\sim 10$ $\mathrm{cm}$.

This short range limited the locations where this coil could be placed. Despite these limitations, the coil in conjunction with the optical link, was able to measure the variation and magnitude of the external magnetic field. These measurements demonstrated the feasibility of magnetic measurements and informed subsequent design iterations.

\section{Version 2: Column-mounted single coil}

The second design iteration uses a smaller coil with about half the cross-sectional area but double the number of turns $(4.2 \times 2.4 \times 0.8 \mathrm{~cm}, N=100, L=\mathrm{mH})$. This provides equivalent sensitivity but can fit into many more locations than the previous coil. The mount is also designed to fit directly onto the heater column such that the coil can be placed closer to the column and ensures the coil area is perpendicular to the azimuthal direction.

The coil contacts are made more robust by attaching the magnet wire leads to twisted-pair gauge 16 wire with a BNC adapter and encasing the connections in hot glue. This ensures the magnet wire is not strained and allows the optical link circuit to be placed further from the coil. Measurements using this sensor identified several oscillation modes in the magnetic field. However, measurements of the the power supply cables indicated that the power supply current oscillates by $\pm 8 \mathrm{~A}$ at $360 \mathrm{~Hz}$. This variation in the total current must be subtracted out to properly measure effects from the kink instability. Consequently, a second sensor is required. 

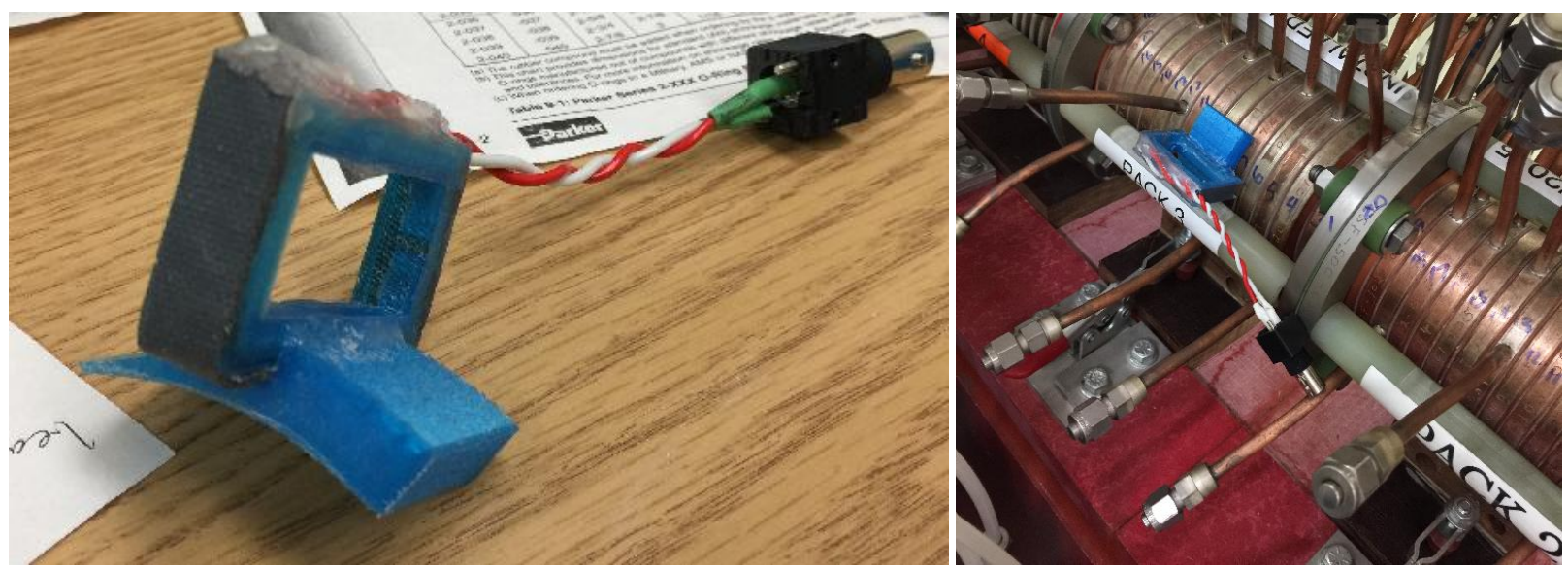

Fig. 6 (Left) Column mounted coil: rectangular coil with 100 turns of AWG 33 wire. 3D printed mount matches curvature of column and thin lower lip fits into the gap between the column and green tension bar. (Right) Coil mounted on Pack 3 of AHF 10 MW heater.

\section{Version 3: Column-mounted differential coil pair}

Placing a pair of coils on opposite sides of the column allows unambiguous measurement of the internal current path motion. The difference between the two signals is proportional to the motion of the internal current path: an increasing signal from sensor \#1 coincident with a decreasing signal from sensor \#2 indicates motion towards sensor \#1. Variations in the total current will affect both sensors equally and do not affect the differential measurement.

The third coil design, shown in Figure 7, altered the base mount slightly to accommodate placement on more packs and provide a better press-fit when attached to the tension bars. This precise fit allows a pair of coils to be accurately placed $180^{\circ}$ apart on opposite sides of the column.
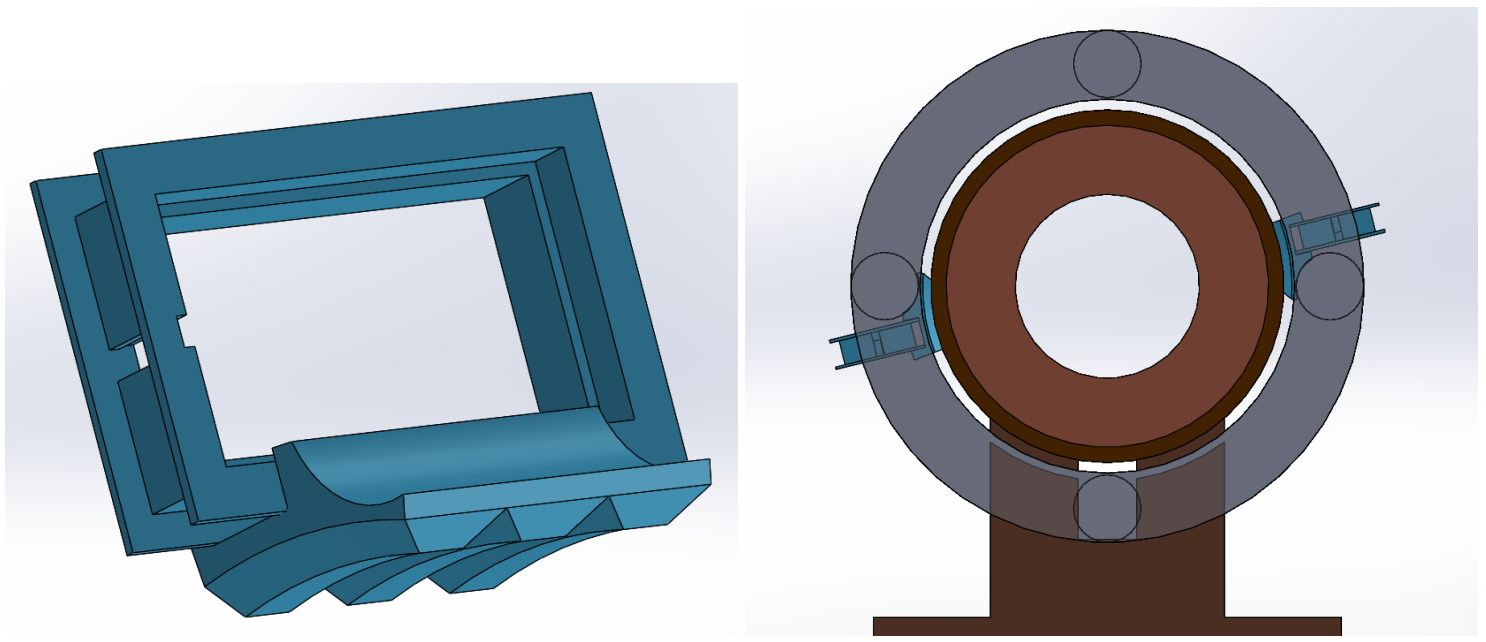

Fig. 7 (Left) Third iteration of coil design: shorter base for easier insertion and larger grooves on base to accommodate boron nitride insulation rings between copper disks. Coil area is the same as in v2: $4.2 \times 2.4 \times 0.8$ cm. (Right) Mounting of coil pair on opposite sides of column. The coils can be accurately placed $180^{\circ}$ apart using the tension bars as mounting points.

A new pair of transmitter/receiver circuit boards with three optical link channels was also constructed to accommodate the second probe and a potential third probe. The three channels on the transmitter and receiver boards share a $5 \mathrm{~V}$ power supply but are otherwise identical to the circuits shown in Figures 2 \& 3 . Channel cross-talk becomes measurable only for frequencies above $1 \mathrm{MHz}$. 


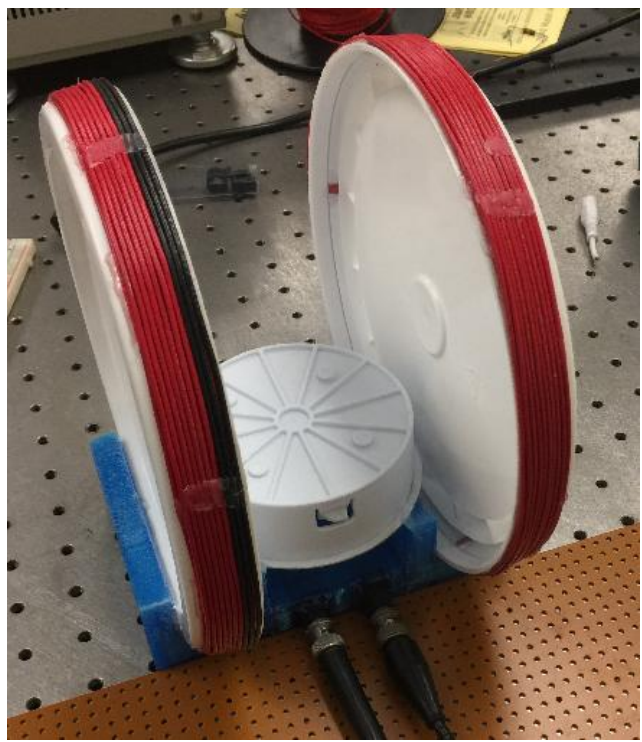

Bdot Probe Calibration

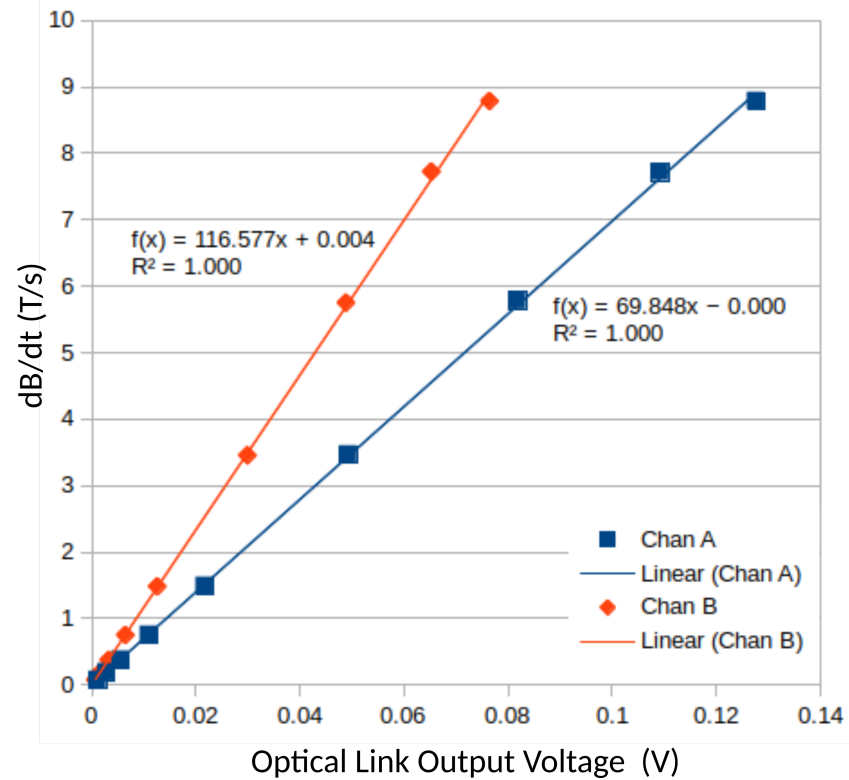

Fig. 8 (Left) Helmholtz coil constructed from bucket lids using 3D printed base: $N=20, R=10.55 \mathrm{~cm}$. Left BNC port attaches to input and output contacts of coils and right BNC port measures voltage across a $2.1 \Omega$ resistor in series with the coils. (Right) Plot of calibration for pair of Bdot probes used in third design iteration.

\section{Calibration}

The coils and optical links were calibrated using the Helmholtz coil shown in Figure 8 (left). The Helmholtz coil consists of two concentric 20 turn circular coils, $R=10.55 \mathrm{~cm}$, spaced one radius apart and connected in series. This arrangement produces a highly uniform magnetic field between the coils which can be used for calibration. The magnetic field can be calculated as

$$
B=\frac{8}{\sqrt{125}} \frac{\mu_{0} N I}{R}
$$

where $N$ is the number of turns in each coil, $R$ is the coil radius, and $I$ is the current. The current through this Helmholtz coil is obtained by measuring the voltage of a $2.1 \Omega$ resistor in series with the coils.

Calibration is conducted on each probe and its associated optical link. The magnetic probe is placed at the center of the Helmholtz coils and attached to the optical link. The Helmholtz coil is driven by a function generator at a particular frequency $f$. An oscilloscope measures the Helmholtz current and output voltage of the optical link. From the Helmholtz current, the applied $B$ is calculated using Eq. 3 and taking the time derivative of this B-field gives $\dot{B}$. It is this time derivative which is then calibrated against the probe output voltage. This process was repeated for a range of frequencies at several different B-field amplitudes (Figure 8, right). The probes behave linearly from $200 \mathrm{~Hz}$ to $100 \mathrm{kHz}$ and for $|\dot{B}|$ in the range of $0-9 \mathrm{~T} / \mathrm{s}$. For the pair of probes in version 3 , the calibration gives,

- Chan A: 69.85 $00.0001(\mathrm{~T} / \mathrm{s})$ per $(\mathrm{V})$

- Chan B: $116.56 \pm 0.004(\mathrm{~T} / \mathrm{s})$ per $(\mathrm{V})$

The significant difference between the two channels is due to differences in the optical links. Since the link circuits use a transistor as a single stage current amplifier, the amplification can differ by a wide range depending on the internal gain of each transistor.

\section{Measurements}

Magnetic data was taken on the AHF (10 MW) column over multiple runs in October and December 2018. The data was recorded using an oscilloscope with limited memory so only small windows of time $(\sim 10 \mathrm{~ms})$ are recorded. 


\section{A. Single coil measurements}

The single coil measurements discovered that the power supply oscillates $\pm 8 \mathrm{~A}$ at $360 \mathrm{~Hz}$. Due to this additional source of magnetic variation, the single coil data could not identify whether the kink instability was occurring in the column. However, the data provided new insight into the behavior of the power supply.

\section{Power supply oscillations}

The Bdot probe was attached to the input current line to directly measure the input current. This input current has a clear oscillating signal at $360 \pm 2 \mathrm{~Hz}$. Figure 9 shows a typical trace and spectrum of the magnetic signal. These oscillations correspond to a \pm 8 A modulation of the input current at $360 \mathrm{~Hz}$. The waveform is not a perfect sine wave so there are additional harmonics at higher frequencies (see Figure 9 , right).
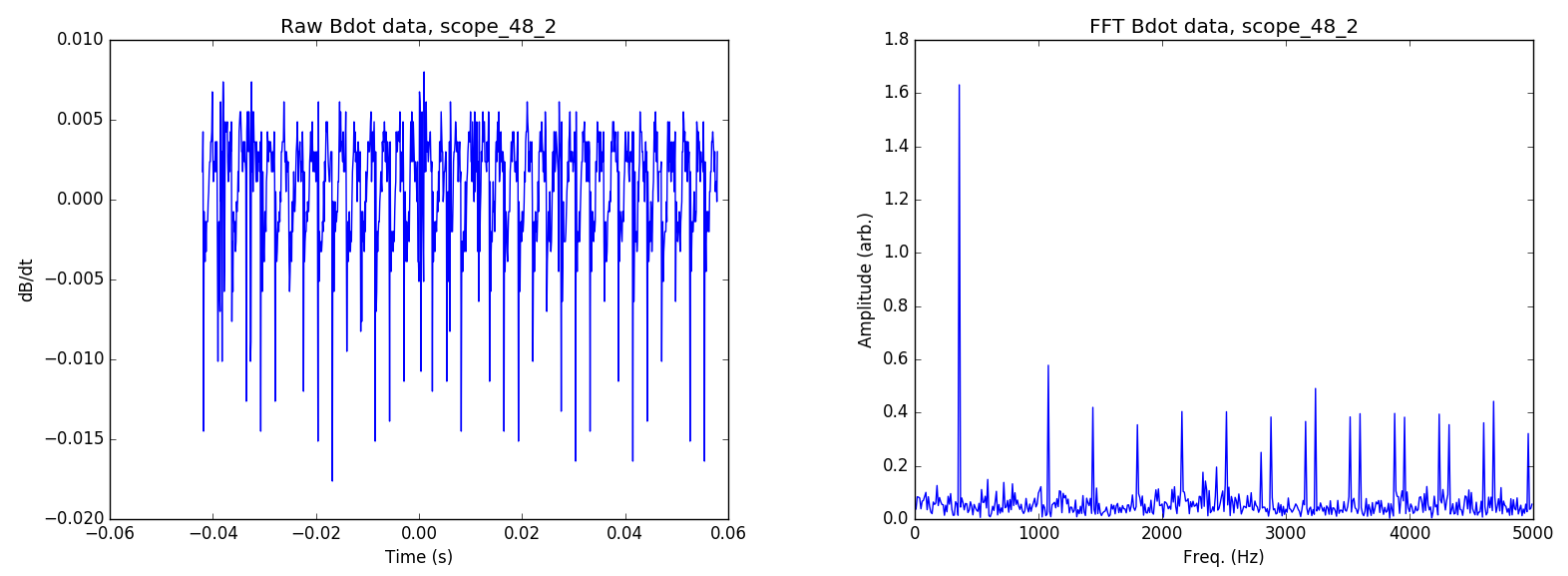

Fig. 9 Plot of raw data collected from the power supply during the 400 A condition on AHF. The FFT shows dominant frequency of $360 \mathrm{~Hz}$ with a number of harmonics.

\section{B. Differential Measurements}

The time dependent displacement of the internal current path was measured using a pair of sensors placed on opposite sides of Pack 10 of the heater column. For a current path with no radial displacement, the two sensors will measure identical signals, even for systems with variable total current. Consequently, the difference in magnitude between the two probes is proportional to the radial displacement of the internal current path.

The relationship between the differential signal $\Delta B$ and the radial displacement $\delta$ is described in the following calculation. The B-field measured by a coil is given by,

$$
\begin{aligned}
\bar{B} & =\frac{1}{r_{2}-r_{1}} \int_{r_{1}}^{r_{2}} \frac{\mu_{0} I}{2 \pi r} d r \\
& =\frac{\mu_{0} I}{2 \pi\left(r_{2}-r_{1}\right)} \ln \left(\frac{r_{2}}{r_{1}}\right)
\end{aligned}
$$

where $r_{1}$ is the inner radius (edge of coil closest to column axis), $r_{2}$ is the outer radius (edge of coil furthest from column axis), $I$ is the total current, and $\bar{B}$ is the measured B-field. Given that the current path is displaced by a distance $\delta$, this will change the relative strength of the B-field at the two probe locations,

$$
\Delta \bar{B}=\bar{B}_{b}-\bar{B}_{a}=\frac{\mu_{0} I}{2 \pi\left(r_{2}-r_{1}\right)}\left[\ln \left(\frac{r_{2}-\delta}{r_{1}-\delta}\right)-\ln \left(\frac{r_{2}+\delta}{r_{1}+\delta}\right)\right]
$$

Solving this equation for $\delta$ gives an expression for the displacement of the internal current in terms of the differential 


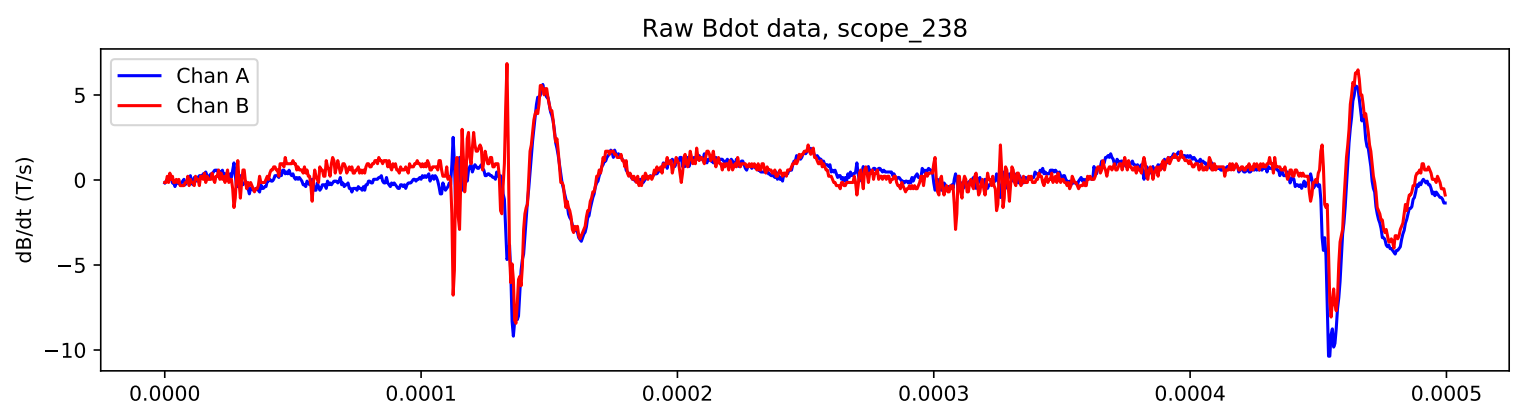

Fig. 10 Plot of typical raw $\dot{B}$ data from differential pair over $0.5 \mathrm{~ms}$. This demonstrates that the high frequency spikes are caused by the power supply rather than motion of the current channel as they produce equivalent signals on both probes.

magnetic field,

$$
\begin{aligned}
& \delta=\frac{\sqrt{4 r_{1} r_{2} \chi^{2}+\left(r_{2}-r_{1}\right)^{2}(\chi+2)^{2}}-\left(r_{2}-r_{1}\right)(\chi+2)}{2 \chi}, \text { where } \\
& \chi=\exp \left(\frac{2 \pi \Delta B\left(r_{2}-r_{1}\right)}{\mu_{0} I}\right)-1
\end{aligned}
$$

\section{Integration Errors}

It is important to quantify integration error since the displacement measurement depends on integrating the $\dot{B}$ values. Similar to how a 1D random walk does not remain at the origin, integration of a signal with some random noise will wander over time. For these magnetic probes, the error associated with this wandering effect is quantified by taking 5 sets of data with the arcjet off, and integrating the base level of noise. This procedure gives integration errors of up to $0.02 \mathrm{G} / \mathrm{ms}$ for the third iteration probes. Since the measured values are on the order of $5 \mathrm{G}$, this error is not significant for these measurements. However, for longer time series ( $>0.50 \mathrm{~s})$, this error could become significant.

\section{L2 Condition}

The L2 condition is a high heat flux condition using $1700 \mathrm{~A}$ and a mass flow of $0.5 \mathrm{~kg} / \mathrm{s}$. This condition typically exhibits large spikes in the $\dot{B}$ signal ( $\pm 8 \mathrm{~T} / \mathrm{s}$ ) with a base variation of $\pm 2 \mathrm{~T} / \mathrm{s}$ (see Figures 10 11). The differential measurements correspond to radial perturbations of $\pm 0.4 \mathrm{~cm}$. The motion has a consistent pattern of fast small displacements at $1-2 \mathrm{kHz}$ (see Figure 12). The longer period displacements at lower frequency are likely driven by the $360 \mathrm{~Hz}$ power supply oscillations.

\section{Conclusions}

The differential magnetic measurements demonstrate that the current channel inside the constrictor is oscillating $\pm 0.4 \mathrm{~cm}$ at $\mathrm{kHz}$ frequencies. This implies that the enthalpy profile of the arcjet experiences similar deviations from the column axis. This motion of the arc has significant consequences for calibration procedures which measure the centerline enthalpy. However, since this effect is produced by magnetic fields, it can also be mitigated by applying additional axial field along the column axis.

Future work will focus on taking 2D measurements of the arc location and developing a suite of Hall sensors to track long-term motions of the arc.

\section{Acknowledgments}

This work was supported by the Entry Systems Modeling (ESM) project (Michael J. Wright, Project manager, and Michael D. Barnhardt, PI) of NASA's Space Technology Mission Directorate. The authors would particularly like to 


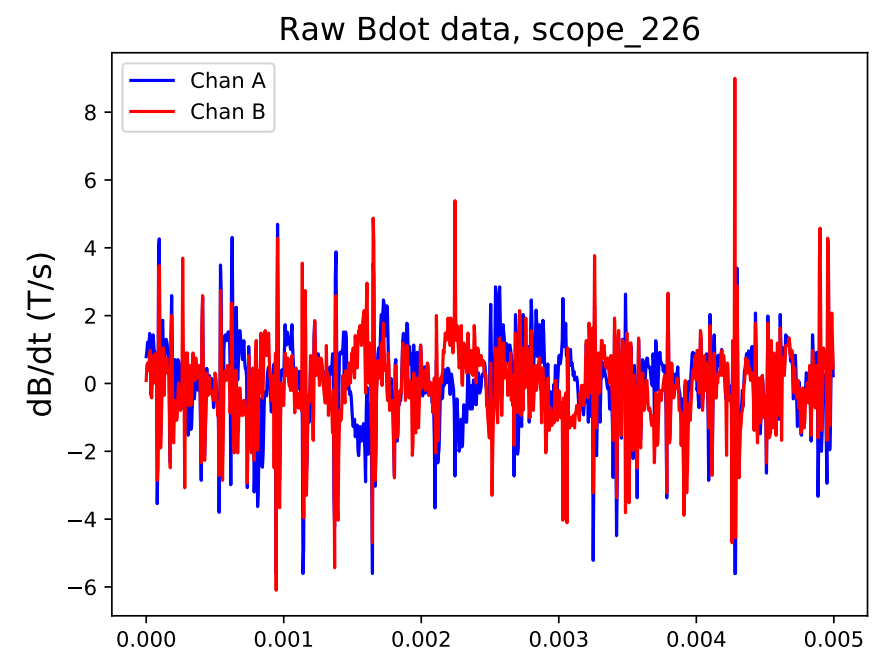

Fig. 11 L2 condition: Raw $\dot{B}$ data for Channels A \& and B are shown for a $5 \mathrm{~ms}$ time window. Several differences between the two channels are visible between 1-3 ms indicating column motion.

thank the arcjet team for their help in setting up measurements and Megan E. Macdonald, Scott G. Eddlemon, Mark McGlaughlin, Ramon Martinez, Rick Ryzinga for providing materials, tools, and advice.

\section{References}

[1] Meurisse, J., Alvarez Laguna, A., and Mansour, N., "3D unsteady model of arc heater plasma flow using the ARC Heater Simulator (ARCHeS),” APS Annual Gaseous Electronics Meeting Abstracts, 2018, p. ET1.009.

[2] Ryutov, D. D., Furno, I., Intrator, T. P., Abbate, S., and Madziwa-Nussinov, T., "Phenomenological theory of the kink instability in a slender plasma column," Physics of Plasmas, Vol. 13, No. 3, 2006, p. 032105. doi:10.1063/1.2180667.

[3] Hsu, S. C., and Bellan, P. M., "Experimental Identification of the Kink Instability as a Poloidal Flux Amplification Mechanism for Coaxial Gun Spheromak Formation,” Phys. Rev. Lett., Vol. 90, 2003, p. 215002. doi:10.1103/PhysRevLett.90.215002, URL https://link.aps.org/doi/10.1103/PhysRevLett.90.215002.

[4] Zhai, X., and Bellan, P. M., "An earth-isolated optically coupled wideband high voltage probe powered by ambient light," Review of Scientific Instruments, Vol. 83, No. 10, 2012, p. 104703. doi:10.1063/1.4757112. 

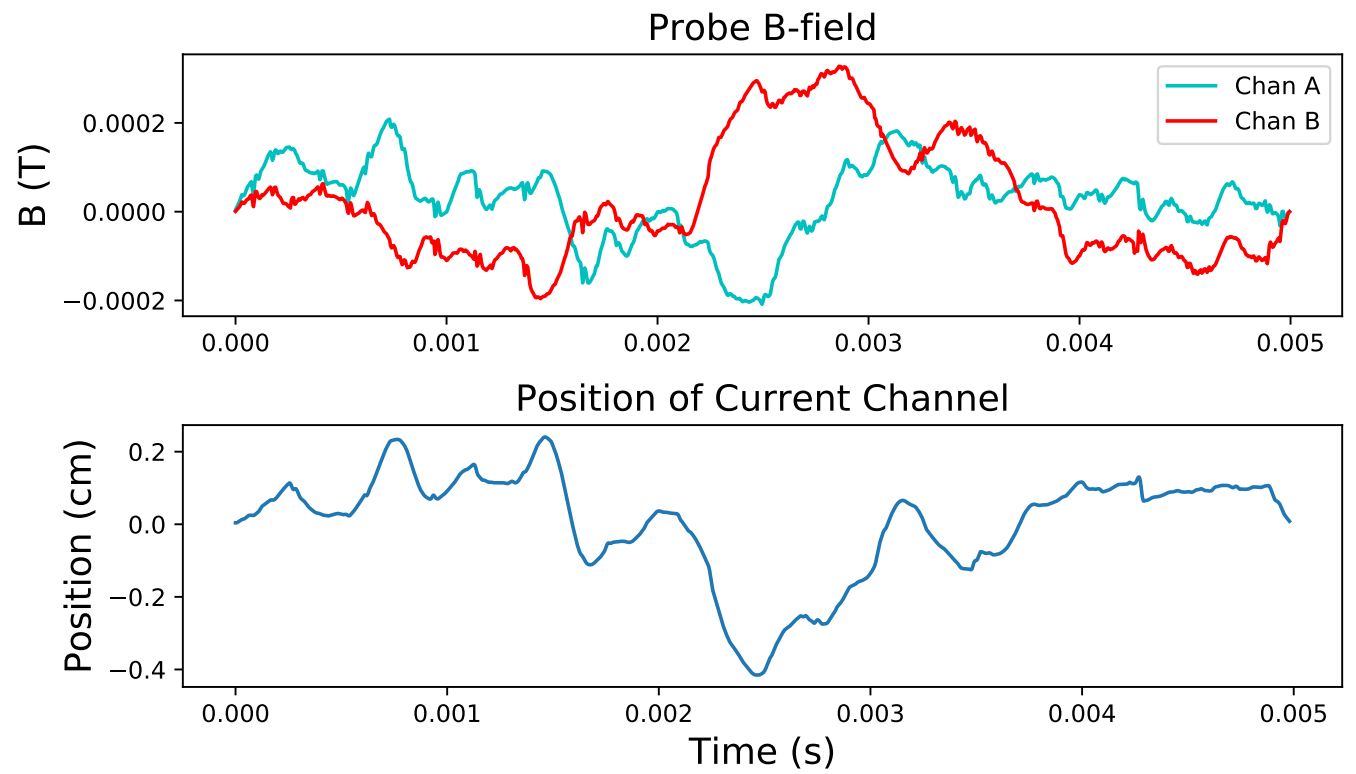

Fig. 12 L2 condition: Plot of integrated B-field at both probes and inferred radial displacement. The displacement shows $\pm 0.4 \mathrm{~cm}$ displacements at $1-2 \mathrm{kHz}$. 